\title{
Successful treatment of pediatric post-liver transplant Kaposi's sarcoma with paclitaxel
}

\author{
Hilal Susam Şen ${ }^{1 \oplus}$, Belen Terlemez Ateş ${ }^{2 \oplus}$, Pınar Yılmazbaş² ${ }^{2 \oplus}$, Süheyla Ocak ${ }^{1 \oplus}$, \\ Hale Kırımlığ̆lü ${ }^{3 \oplus}$, Selim Gökçe ${ }^{4 \oplus}$, Koray Acarlı ${ }^{5 \oplus}$ \\ ${ }^{1}$ Division of Pediatric Oncology, ${ }^{2}$ Department of Pediatrics, Health Sciences University, Okmeydanı Training and Research Hospital, \\ Istanbul; ${ }^{3}$ Department of Pathology, Acıbadem University School of Medicine, İstanbul; Departments of ${ }^{4}$ Pediatric Gastroenterology and \\ ${ }^{5}$ Pediatric Surgery and Liver Transplantation Center, Memorial Şişli Hospital, İstanbul, Turkey.
}

\begin{abstract}
Background. Kaposi's sarcoma (KS) is a complication of immunosuppressive therapy for transplant recipients. Unlike adult recipients, KS in pediatric organ transplantation is quite rare. Treatment is usually withdrawal of immunosuppression; non-responders often receive chemotherapy.
\end{abstract}

Case. We have reported a child with post-liver transplant visceral KS which has progressed despite withdrawal of immunosuppressive therapy, who has been treated with Paclitaxel for three weeks. KS has regressed completely after four cycles of Paclitaxel.

Conclusion. Paclitaxel should be considered as an effective first line treatment option for patients with posttransplant KS.

Key words: Kaposi's sarcoma, children, paclitaxel.

Kaposi's sarcoma (KS) is a rare type of visceral and soft tissue sarcoma related to human herpes virus (HHV)-8 characterized by the proliferation of spindle shaped cells, mainly of vascular origin. ${ }^{1}$ Clinically, KS is a slowly progressing malign lesion commonly manifesting with cutaneous lesions with or without involvement of internal visceral organs and/or lymph nodes. ${ }^{1}$ It is classified clinicopathologically into four subgroups based on epidemiological data which are classical, endemic (African type), epidemic (AIDS-related), and iatrogenic (transplantation-related) KS. The type observed in the organ transplant recipients is known as a type that is considered to be related to immunosuppression and may regress after the discontinuation of the therapy or may show an aggressive and multifocal course compared to classical type. ${ }^{2}$ Both sporadic and opportunistic

$凶$ Hilal Susam Şen

hilalsusam@hotmail.com

Received 17th December 2019,

accepted 15th February 2020. forms of KS are associated with the presence of the HHV-8 genome in the neoplastic cells. Although its incidence varies across different types, the most common presentation is purple, blue, red or dark brown, black macula, papule, plaque and nodular skin lesions and accompanying lymphedema, especially in the lower extremities. ${ }^{1}$ Systemic therapy may be considered in the patients with symptomatic visceral and mucosal involvement. For systemic therapy, in addition to the drugs such as anthracyclines, vinca alkaloids, bleomycin, oral etoposide and gemcitabine, taxanes have also being used during recent years for their antiangiogenic effects.,4 Moreover, several studies have shown that paclitaxel monotherapy is a successful second-line treatment for both AIDS-associated KS and KS in a therapeutically immunosuppressed patient. ${ }^{4}$ Additionally, for patients with epidemic or iatrogenic KS, the most effective treatment requires correcting the underlying immunodeficiency. ${ }^{2}$

In this report, we present a patient with disseminated visceral KS after liver 
transplantation who has showed a dramatic response to Paclitaxel therapy.

\section{Case Report}

A seven year-old girl with chronic liver failure secondary to progressive familial intrahepatic cholestasis type III (PFIC III) was transplanted with a left lateral segment from her mother. The patient was third of four children from a first degree-cousin marriage. Her older brother and cousin were also diagnosed with PFIC III, and both were liver transplanted. According to current immunosuppression protocol, she received IV methylprednisolone at the dose of $10 \mathrm{mg} / \mathrm{kg}$ during surgery and the dose was decreased to $2 \mathrm{mg} / \mathrm{kg} /$ day at the end of the first week. Then, steroid was tapered to the dose of $0.5 \mathrm{mg} / \mathrm{kg} /$ day in the post-transplant first month and withdrawn at the end of the post-transplant third month. She was given tacrolimus during the post-transplant period. One year after transplantation, she was presented with ascites. On physical examination of the oral cavity, there were $2 \times 3 \mathrm{~cm}$ brown-blue-red nodule posterior of lower incisors (Fig. 1). Complete blood count revealed pancytopenia. Coagulation profile together with blood biochemistry was unremarkable. Serological tests for CMV and EBV IgM, CMV antigen testing, PCR for CMV and EBV were all negative. In addition, HIV antigens were negative. Abdominal CT revealed multiple enlarged mesenteric lymph nodes.

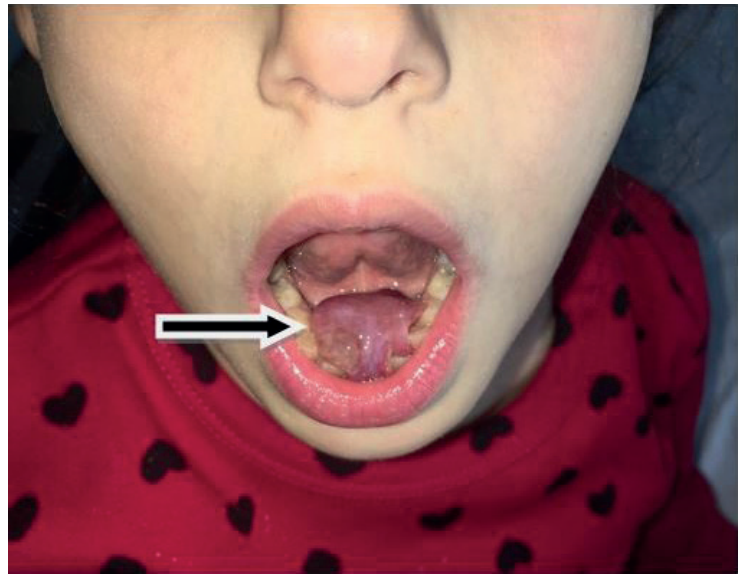

Fig. 1. Brown-blue-red nodule in the oral cavity.
Endoscopic examination revealed multiple polypoid brown-blue-red lesions on esophagus, stomach and duodenum. Multiple biopsies from said lesions revealed an infiltration of neoplastic cells with round-oval nuclei and moderatesized eosinophilic cytoplasm, and red blood cells among them. In immunohistochemical assessment, the neoplastic cells displayed CD34, CD31 (Fig. 2a) and HHV-8 immunoreactivity (Fig. 2b). The histopathological examination of biopsy was consistent with HHV-8 related KS. Tacrolimus was switched to sirolimus at a dose of $2 \mathrm{mg} / \mathrm{m}^{2}$ to keep the serum levels around $5 \mathrm{ng} / \mathrm{ml}$. In due course, no regression of the lesions was observed. Paclitaxel therapy at a dose of $100 \mathrm{mg} / \mathrm{m}^{2}$ per three weeks was started. ${ }^{5}$ Informed consent was received from the family. Oral lesions regressed rapidly following first cycle; after four cycles of paclitaxel, all lesions were resolved completely (Fig. 3). The gastrointestinal endoscopic examination was macroscopically absent of any kaposiform lesion and histopathology of multiple biopsies was completely normal. Paclitaxel was tolerated well by the patient. Bone marrow suppression which developed during therapy was easily controlled by supportive therapy without any clinical manifestation. Now the patient is free of disease for at least 18 months and completely healthy with good hepatic graft function.

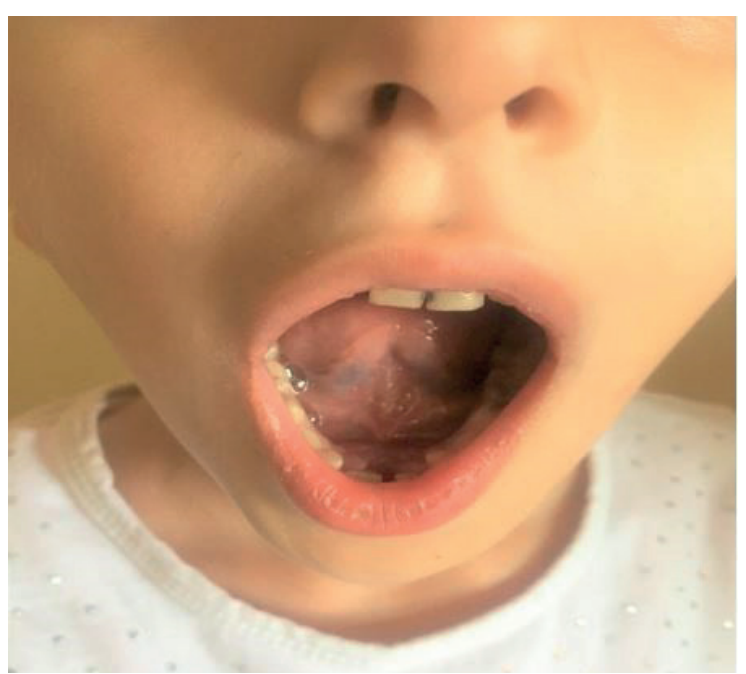

Fig. 3. After four cycles of paclitaxel therapy, the lesion in the oral cavity regressed totally. 

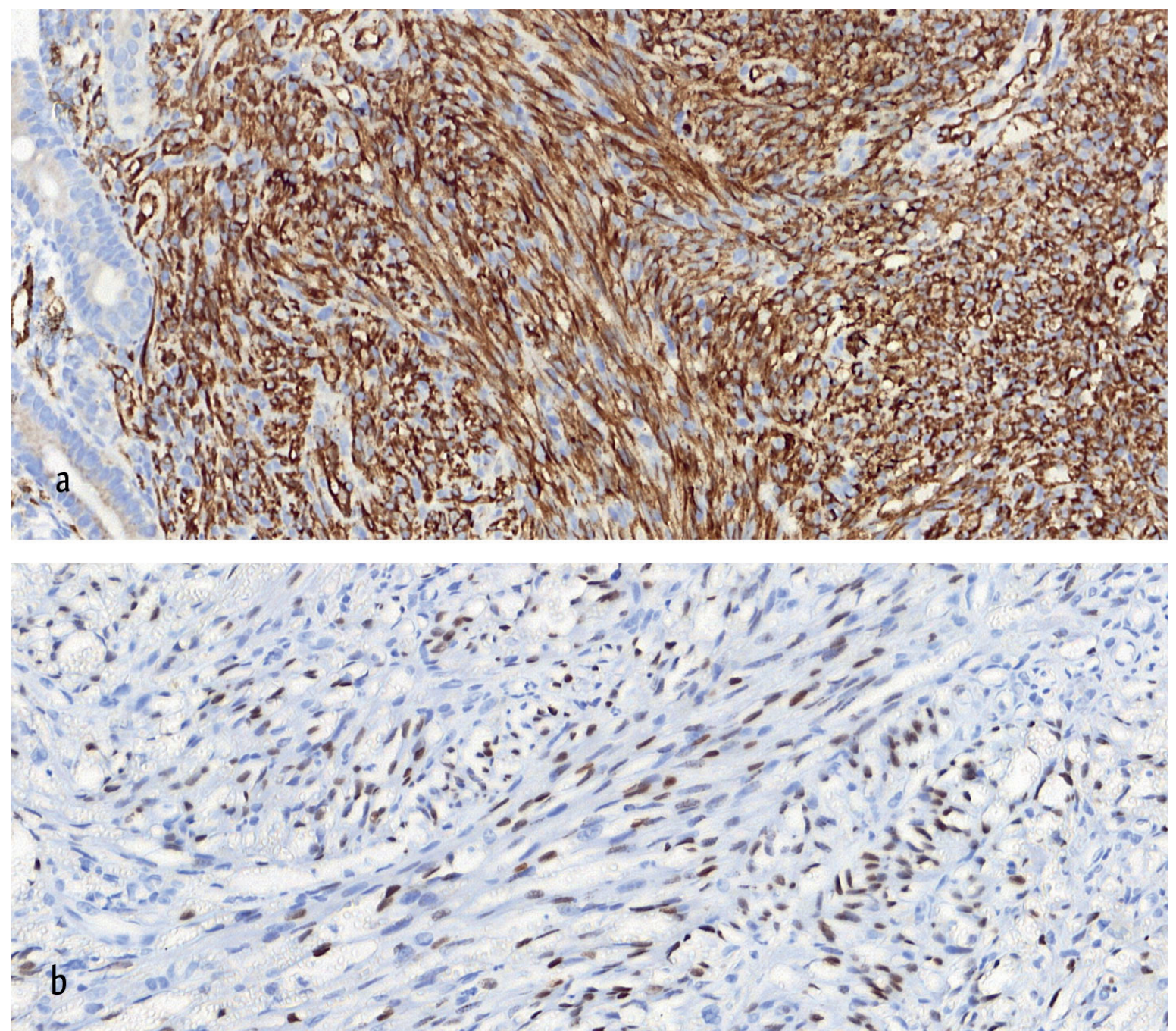

Fig. 2. Immunohistochemical staining of biopsy specimens. Figure $2 \mathrm{a}$ and $2 \mathrm{~b}$ show CD31 positivity and HHV-8 immunoreactivity, respectively.

\section{Discussion}

Because of the rarity of pediatric KS, there is a paucity of literature on treatment and outcome stratification with most treatment modality options extracted from literature of adults treated for KS. To date, there is no established consensus group therapeutic guidelines for the treatment of all four forms of pediatric KS.

Strong correlation between the immunosuppressive status of the patient and the development of KS is very well demonstrated, and it is now a major and increasing problem in organ transplant recipients. ${ }^{6}$ Unlike adult recipients, KS in pediatric organ transplantation is quite rare and mainly observed in renal allograft recipients. ${ }^{6}$ It is reported rarely after liver transplantation in children.7,8 Compared with renal transplant recipients, visceral involvement is more frequent among liver transplant recipients. ${ }^{9}$ So, the tendency to dissemination and systemic nature of $\mathrm{KS}$ in liver transplant recipients is considered to be responsible for shorter survival than those who have undergone renal transplantation. ${ }^{10}$

Reduction or discontinuation of immunosuppressive treatment is the first step in the treatment of iatrogenic KS and it has been associated with the disappearance of lesions. ${ }^{11,12}$ 
In addition, there is evidence that patients with iatrogenic KS have had tumor regression when immunosuppression has been switched to sirolimus, a mammalian target of rapamycin (mTOR) inhibitor sharing both immunosuppressive and anti-neoplastic effects. ${ }^{13}$ Unlike other immunosuppressants, sirolimus has potent antitumor activity in vitro and in vivo. Sirolimus having antiproliferative and antitumor effect has been increasingly reported in the management of the disease.,9 In the presented case, tacrolimus has been withdrawn and sirolimus has been started. However, lesions have remained unaltered and chemotherapy has commenced.

Therapeutic options for KS are based upon disease stage, progression pattern and distribution, clinical type, and immune status. ${ }^{14}$ For KS patients with more widely disseminated, progressive or symptomatic disease, systemic therapy with cytotoxic chemotherapy is generally warranted. The present case was not eligible for local therapy because she had multiple visceral and mucosal lesions. Several single agent therapies have been reported to be active in all subtypes of KS (vincristine, vinorelbine, etoposide, adriamycin, liposomal doxorubicin, epirubicin, bleomycin, docetaxel and paclitaxel) with an overall response rate ranging from 30 to $70 \%$, although most of them have been partial responses. ${ }^{3}$

Taxanes (paclitaxel and docetaxel) are now promising agents in the treatment of KS with tolerable side effects. They have potent antiangiogenic activities, which may explain their efficacy on Kaposi lesions. ${ }^{15}$ Paclitaxel is a drug affecting the microtubules and cellular vital processes in nonmitotic phases of the cell cycle and it inhibits the growth of proliferating tumors either rapidly or slowly. ${ }^{14}$ The efficacy of paclitaxel given as second-line or third-line therapy has been evaluated well in association with highly active antiretroviral therapy in patients with AIDS-related KS. ${ }^{16,17}$ Recently, some articles about its efficacy in the treatment of classical, endemic and post-transplantation types of KS have also been published..$^{18}$ Patel et al. ${ }^{5}$ have demonstrated prompt and durable response in two renal transplant recipients.

Limited cases of pediatric iatrogenic KS have shown promising response to MTOR inhibitor sirolimus, and paclitaxel chemotherapy.,8 Based on these data, because of promising results and tolerable side effects, we decided to start paclitaxel monotherapy as first line treatment. We continued to give sirolimus after the completion of chemotherapy regimen as maintenance, because it is an effective agent in both treatments of KS and prevention of organ rejection.

Pediatric patients with post-liver transplant who develop KS may face a dismal prognosis. ${ }^{8,19}$ Celtik et al. ${ }^{8}$ describe a 15 -month-old child who underwent liver transplant with subsequent pancytopenia, lymphadenopathy, edema, and subconjunctival bleeding. A cervical lymph node biopsy confirmed the diagnosis of disseminated KS. The first chemotherapy choice was weekly vinblastine, but the child was unresponsive. Second-line chemotherapy with, actinomycin D and cyclophosphamide was not effective. Then durable response was ensured by paclitaxel. Similar findings of multi-visceral KS after pediatric liver transplant have also been described in Mexico, France, and Turkey; many with fatal outcomes. ${ }^{719}$ Anthracyclines are another treatment option but limited effects with cardiotoxic side effects restrict its preference. ${ }^{3}$ When evaluating child and adult data on posttransplant KS, cytotoxic chemotherapy does not appear to be very effective. In addition, serious side effects associated with these treatments have been observed. Recently, efficacy of paclitaxel in post-transplant type KS has been published. ${ }^{5}$ In a review of Dow et al. ${ }^{20}$ paclitaxel was the first chemotherapy option in pediatric post-transplant KS. Paclitaxel-related side effects were also much fewer than other chemotherapeutic agents. ${ }^{4}$

In the present case, KS regressed completely after four cycles of Paclitaxel and remained in remission for 18 months. 
In conclusion, $\mathrm{KS}$ continues to cause significant morbidity and mortality worldwide in both pediatric and adult populations. Our experience suggests that paclitaxel is a promising and effective first line option for treatment of iatrogenic KS with tolerable side effects and sustained response. Further studies are required to standardize the paclitaxel treatment schedule and dosage in the disseminated iatrogenic type of KS.

\section{REFERENCES}

1. Schneider JW, Dittmer DP. Diagnosis and treatment of Kaposi sarcoma. Am J Clin Dermatol 2017; 18: 529-539.

2. Jemal A, Bray F, Center MM, Ferlay J, Ward E, Forman D. Global cancer statistics. CA Cancer J Clin 2011; 61: 69-90.

3. Stewart S, Jablonowski H, Goebel FD, et al. Randomized comparative trial of pegylated liposomal doxorubicin versus bleomycin and vincristine in the treatment of AIDS-related Kaposi's sarcoma. International Pegylated Liposomal Doxorubicin Study Group. J Clin Oncol 1998; 16: 683-691.

4. Gill PS, Tulpule A, Espina BM, et al. Paclitaxel is safe and effective in the treatment of advanced AIDSrelated Kaposi's sarcoma. J Clin Oncol 1999; 17: 1876-1883.

5. Patel N, Salifu M, Sumrani N, et al. Successful treatment of post-renal transplant Kaposi's sarcoma with paclitaxel. Am J Transplant 2002; 2: 877-879.

6. Debray D, Baudouin V, Lacaille F, et al; Pediatric Transplantation Working Group of the French Speaking Society of Transplantation. De novo malignancy after solid organ transplantation in children. Transplant Proc 2009; 41: 674-675.

7. Yuksekkaya HA, Arikan C, Yazici A, Baran M, Aydogdu S, Kilic M. Successful treatment of a child having generalized Kaposi's sarcoma after living donor liver transplantation with conversion to sirolimus. Pediatr Transplant 2009; 13: 375-378.
8. Celtik C, Unüvar A, Aydoğan A, et al. Human herpes virus type 8-associated Kaposi sarcoma in a pediatric liver transplant recipient. Pediatr Transplant 2011; 15: E100-E104.

9. Guba M, Yezhelyev M, Eichhorn ME, et al. Rapamycin induces tumor-specific thrombosis via tissue factor in the presence of VEGF. Blood 2005; 105: 4463-4469.

10. Aseni P, Vertemati M, Minola E, et al. Kaposi's sarcoma in liver transplant recipients: morphological and clinical description. Liver Transpl 2001; 7: 816823.

11. Penn I. Sarcomas in organ allograft recipients. Transplantation 1995; 60: 1485-1491.

12. Francès C. Kaposi's sarcoma after renal transplantation. Nephrol Dial Transplant 1998; 13: 2768-2773.

13. Stallone G, Schena A, Infante B, et al. Sirolimus for Kaposi's sarcoma in renal-transplant recipients. N Engl J Med 2005; 352: 1317-1323.

14. Tschachler E. Kaposi sarcoma. In: Wolff K, Goldsmith LA, Katz SI, Gilchrest BA, Paller AS, Leffell D, (eds). Fitzpatrick's Dermatology in General Medicine (7th ed). New York: McGrow-Hill, 2008: 1183-1187.

15. Vacca A, Ribatti D, Iurlaro M, et al. Docetaxel versus paclitaxelfor antiangiogenesis. J Hematother Stem Cell Res 2002; 11: 103-118.

16. Saville MW, Lietzau J, Pluda JM, et al. Treatment of HIV-associated Kaposi's sarcoma with paclitaxel. Lancet 1995; 346: 26-28.

17. Von Roenn JH, Krown SE. Management of AIDSassociated Kaposi's sarcoma: a multidisciplinary perspective. Oncology (Williston Park) 1998; $12(2$ Suppl 3): 1-24.

18. Chao SC, Lee JY, Tsao CJ. Treatment of classical type Kaposi's sarcoma with paclitaxel. Anticancer Res 2001; 21: 571-573.

19. Marcelin AG, Roque-Afonso AM, Hurtova M, et al. Fatal disseminated Kaposi's sarcoma following human herpesvirus 8 primary infections in livertransplant recipients. Liver Transpl 2004; 10: 295300.

20. Dow DE, Cunningham CK, Buchanan AM. A review of human herpesvirus 8, the Kaposi's sarcomaassociated herpesvirus, in the pediatric population. J Pediatric Infect Dis Soc 2014; 3: 66-76. 